\title{
SUPEROXIDE DISMUTASE LOADED NIOSOMES DELIVERY TO HAIR FOLLICLES: PERMEATION THROUGH SYNTHETIC MEMBRANE AND GUINEA PIG SKIN
}

\author{
MASOUD ALI KARAMI², MARZIE JALILI RAD ${ }^{2}$, BEHZAD SHARIF MAKHMAL ZADEH ${ }^{1,2^{*}}$, ANAYATOLLAH SALIMI1,2
}

${ }^{1}$ Nanotechnology Research Center, Ahvaz Jundishapur University of Medical Sciences, Ahvaz, Iran, ${ }^{2}$ School of pharmacy, Ahvaz Jundishapur University of Medical Sciences, Ahvaz, Iran

Email: makhmalzadeh@yahoo.com

Received: 24 May 2019, Revised and Accepted: 06 Aug 2019

\section{ABSTRACT}

Objective: Alopecia aretea is associated with an increase in free radicals causing damage to hair follicles. Superoxide dismutase (SOD) with sufficient penetration through hair follicles, can prevent their death by its strong antioxidant effects. SOD with high molecular weight underwent limitation in follicular delivery. The aim of this study was the improvement of SOD localization into hair follicles.

Methods: SOD-loaded niosomes were prepared by thin layer hydration method and were used as a vehicle for delivery to hair follicles through guinea pig skin and the synthetic membrane. Particle size, entrapment efficiency, drug release, and permeability parameters through hairly and non-hairly pig skin compared with a synthetic membrane were evaluated.

Results: Niosomes demonstrated 152-325 nm particle size and the SOD burst and sustained release from niosomes were mainly controlled by diffusion and dissolution phenomena. SOD was protected against degradation by niosomes and after six months, enzyme content and activity decreased less than $5 \%$. In comparison with free SOD, niosomes increased SOD affinity to penetration through follicles by interaction with sebum. Likewise, niosome's characters such as type of surfactant, solid lipid/liquid lipid ratio played critical roles on SOD deposition on hair follicles.

Conclusion: Synthetic membrane and hairy guinea pig skin demonstrated similar barrier property against free-SOD thereby implying that free SOD does not interact with guinea pig sebum. Niosomes can introduce a suitable carrier for SOD localization into the hair follicles.

Keywords: Superoxide dismutase, Niosome, Hair growth, Alopecia areata, Follicular drug delivery

(C) 2019 The Authors. Published by Innovare Academic Sciences Pvt Ltd. This is an open access article under the CC BY license (http://creativecommons.org/licenses/by/4.0/) DOI: http://dx.doi.org/10.22159/ijap.2019v11i5.34289

\section{INTRODUCTION}

Alopecia areata (AA) isa hair loss disease resulting from an inflammatory, autoimmune disorder that is associated with damage to hair follicles. AA is accompanied by an increase in free radicals such as reactive oxygen species (ROS) and nitric oxide; and a decrease of the antioxidant activity that is provided by enzymes such as superoxide dismutase (SOD). Along the course and increased severity of the disease, protective mechanisms of antioxidant enzymes will be inadequate thereby resulting in oxidative damage to cellular components [1-6]. SOD has an important role in the defense against free radical damage that catalyzes the highly reactive dismutation superoxide anion to oxygen and the less reactive hydrogen peroxide [7].

SOD also helps to carry nitric oxide to the hair follicles which by acting as a vasodilator benefits hair follicles by allowing increased blood flow [8].

Targeted SOD delivery may enhance therapeutic approaches to the treatment of AA. Bulge region and follicular papilla were introduced as two major targets in hair follicles for AA treatment [9]. Drug delivery to these sites may be limited by structural, physical and chemical barriers [10]. Penetration through hair follicles is highly dependent upon the hydrophilic and lipophilic properties and particle size of vehicles and can be improved by using suitable vehicles. Emerit et al. showed that SOD applied to the skin surface penetrates through the follicular appendages as well as the unbroken stratum corneum [11]. SOD, however, is a large molecule with high aqueous solubility and cutaneous absorption is a major concern when it is considered for topical treatment [12]. In recent years many efforts have been made to enhance drug deposition to hair follicles using different drug delivery systems such as niosomes [13]. Niosomes are non-ionic surfactant based vesicle that demonstrating a slow pattern of release [14]. Niosomes have several advantages such as low cost, great chemical stability, high entrapment capacity and low particle size that could provide increased drug permeation and localization into the skin [15-18].
Tabakhian and his colleagues have studied the in vitro and in vivo skin application of finasteride loaded in niosomes to increase drug concentration in pilosebaceous [19].

Manosroi and colleagues reported that optimal follicular delivery was occurred by niosomes prepared by poly-unsaturated fatty acid with zeta potential around-36 mV and particle size of about $200 \mathrm{~nm}$ [14].

A number of studies have evaluated the difference between follicle-rich versus follicle-free skin models using both hairy and hairless animal skin. Human skin availability is limited and so animal models such as a mouse, rat, pig, guinea pig, and snake have been used as an alternative in follicular drug delivery studies [20]. Because of availability, low cost and ease of handling, mouse, rat, and guinea pig skin are the most commonly used in in vitro and in vivo percutaneous absorption studies [21] Anderson et al. evaluated percutaneous absorption of [14] C ring-labeled hydrocortisone, testosterone, and benzoic acid through guinea pig skin and compared the results with previous human data [22]. They reported that the absorption of hydrocortisone and benzoic acid was similar to human skin; however, testosterone was absorbed in a greater rate in guinea pigs compared to humans. Synthetic membranes are used as models for follicular and transdermal absorption and demonstrated several advantages $[23,24]$.

In this study, we followed two main aims. At first, the aim of this research was an evaluation of the ability of niosomes for site-specific delivery of SOD into hairy skin. Secondly, we tried to introduce synthetic membrane as follicular rout imitation model. Permeability studies with synthetic membranes instead of in vivo and in vitro experiments through human and animal skins may overcome the problems associated with these studies.

\section{MATERIALS AND METHODS}

\section{Materials}

Zn, Cu-SOD from bovine erythrocytes, SOD assay kit, and Bradford reagent were purchased from Sigma-Aldrich (USA). Cholesterol, egg lecithin, oleic acid, and sodium lauryl sulfate (SLS) were provided from 
Sigma-Aldrich and oleoyl macrogol-6 glycerides: Labrafil SM 1944 C® (Labrafil) was gifted from Gattefosse [France). Methanol and chloroform were purchased from Merck (Germany). Bovine serum albumin (BSA) was purchased from Sigma-Aldrich. All other materials and solvents used in this study were of analytical grade. The synthetic membrane was made by Dorsa Behsaz Co (Tehran, Iran). For this purpose, human scalp hairs including 90 hair follicles with $45 \mu \mathrm{m}$ per $\mathrm{cm}^{2}$ such as actual human condition obtained from females were fixed in a chamber and filled with resin at room temperature.

\section{Animals}

Female adult guinea pigs ranging in size from $600-800 \mathrm{~g}$ with the age of 20-22 w were purchased from Razi Institute (Iran). Animals were kept for $5 \mathrm{~d}$ of acclimatization, and given a standard diet and water and were then treated according to the principles for the care and use of laboratory animals. Approval for the studies was given by the Ethical Committee of the Ahvaz Jundishapur University of Medical Sciences (approval number: N-104). The guidelines followed were those laid down by the National Academy of Sciences and published by the National Institutes of Health (U. S. Department of Health and human services, Office of laboratory animal welfare).

\section{Experimental design for preparation of niosomes}

Several parameters influence on final properties of niosomes and permeation through hair follicles. The full-factorial design was used concerning with 3 variables at 2 levels as experimental design in this study. Solid lipid/liquid lipid w/w ratio (S. L/l. L), lipid/surfactant $\mathrm{w} / \mathrm{w}$ ratio (L/S) and type of surfactant (T. S) were chosen as independent variables (table I). Cholesterol and oleic acid were used as solid and liquid lipids, respectively. Labrafil as non-ionic and sodium Lauryl sulfate as an anionic surfactant was chosen for the evaluation of the effect of charge on the follicular drug delivery ability of niosomes.

Independent parameters and their levels were chosen based on the preformulation study. Dependent variables include SOD entrapment efficiency, noisome size, drug release and permeation parameters through guinea pig skin and synthetic membrane. The effects of independent variables on dependent variables were evaluated by calculation of correlation coefficient $\left(\mathrm{r}^{2}\right)$ (explained variation/total variation), adjusted $\mathrm{r}^{2}$ (one type of $\mathrm{r}^{2}$ that evaluates regression model's explanatory power) and F-test (in regression compares the fits of different linear models). The intensity of variables interaction on each response was estimated through simultaneous multiple regression with Minitab 16 software. A total of eight experimental runs were generated for the evaluation of the main effects and possible interactions. Accordingly, eight samples, with $0.5 \% \mathrm{~W} / \mathrm{V}$ surfactan 1 or surfactant2, with $8 \mathrm{mg}$ of cholesterol as solid lipid, and varying in the amount of oleic acid (0.21-0.44 mg) and lecithin (0.21-0.44 $\mathrm{mg}$ ) as liquid lipids were prepared (formulations F1-8, table II).

Table 1: Independent variables considered in experimental design and their levels

\begin{tabular}{lll}
\hline Independent variable & Low level & High level \\
\hline S. L/l. L & 9 & 19 \\
L/S & 10 & 20 \\
T.S & Labrafil & Sodium lauryl sulfate \\
\hline
\end{tabular}

\section{Niosome preparation}

Niosomes were prepared by the thin-film hydration method. Accurately weighed quantities of the surfactant (SLS or Labrafil), cholesterol, lecithin, and oleic acid were dissolved in chloroform and methanol (2:1) in a round bottom flask. The organic solvents were removed under vacuum in a rotary (IKA RV05, Germany) evaporator at $55^{\circ} \mathrm{C}$ for $30 \mathrm{~min}$ and $200 \mathrm{rpm}$ to form a thin film on the wall of the flask. This was kept under vacuum for $2 \mathrm{~h}$ to ensure total removal of trace solvents. After $2 \mathrm{~h}$, the dry lipid film was hydrated with $10 \mathrm{ml}$ of SOD solution (50 Unit/ml in phosphate buffer, $\mathrm{pH}$ 7.4) with the resulting dispersion undergoing sonication in a bath-type sonicator (T-710 DH-ELMA, Germany) in 3 cycles of $3 \mathrm{~min}$ "on"/5 min "off" leading to the reduction in particle size of formed niosomes [25].

\section{SOD activity assay}

The activity of SOD was determined by the ability of SOD in converting superoxide radicals to hydrogen peroxide that causes inhibition in indicator oxidation and in result inhibits color formation [26].

\section{Protein assay method}

Total protein concentration was determined by Bradford method with BSA as standard protein.

\section{SOD entrapment efficiency (EE\%)}

The amount of SOD entrapped in the niosomes was measured by an indirect method. In the indirect method, the calculation was done based on the difference between the total amount of SOD applied in the niosomal formulation and the amount of non-entrapped SOD remaining in the supernatant. SOD niosomal formulations were centrifuged at $15700 \times \mathrm{g}$ for $60 \mathrm{~min}$ at $4{ }^{\circ} \mathrm{C}$, to separate the supernatant including the unloaded enzyme. SOD concentration in the supernatant was determined with a UV spectrophotometer at $595 \mathrm{~nm}$ by the Bradford method. The percentage of SOD entrapped in the niosome calculated by the following equation. This process was repeated thrice to completely free SOD was removed [27].
$\%$ drug entrapment $=\left[\frac{\text { total drug }- \text { drug in spernatant }}{\text { total drug }}\right] \times 100$

\section{Particle sizes and zeta potential measurement}

The mean size of niosomes was determined at $25^{\circ} \mathrm{C}$ by laser light diffractometry using Malvern Zetasizer Nano ZS (Malvern instrument, UK). Each niosomal formulation was diluted (20x) with phosphate buffer ( $\mathrm{pH}$ 7.4) before the experiment was carried out. Each sample was measured five times. The zeta potential of niosomes was determined using phase analysis light scattering. Sample preparation was the same as for particle size measurements. The length of time of analysis was 60 seconds. Each sample was measured five times.

\section{Atomic force microscopy (AFM)}

On the other hand, AFM visualization (LEO, Germany) presents information about the shape and size of niosomes. For this purpose, Atomic Force Microscopy (JPK, Nanowizaerd II, Germany) was used. $5 \mu \mathrm{l}$ of the niosome solution was loaded in the center of a split mica disk. The image was set to tapping mode and the average scanning speed was $5 \mathrm{~Hz}$.

\section{Physical stability of niosomes}

All formulations were stored at $2-8{ }^{\circ} \mathrm{C}$ for 6 mo and niosome size, Zeta potential, enzyme activity and protein content, appearance, and drug release were assessed. The results were correspondingly compared with the values of freshly prepared samples.

\section{In vitro release studies}

In vitro SOD release was assessed by the dialysis technique. For each formulation, $10 \mathrm{ml}$ was centrifuged at $15700 \times \mathrm{g}$ for $25 \mathrm{~min}$ at $4{ }^{\circ} \mathrm{C}$ to separate niosomes. The pellet was washed twice with water, centrifuged, dried and then suspended in $5 \mathrm{ml}$ of distilled water. The niosome suspension was then placed in cellulose acetate dialysis tubes (10 kDa, Sigma-Aldrich) which were immersed in $200 \mathrm{ml}$ of phosphate buffer ( $\mathrm{pH} 7.4)$ at $37^{\circ} \mathrm{C}$ that was magnetically stirred at 
$50 \mathrm{rpm}$. At predetermined time intervals $(0.5,1,2,3,4,5,6,7,8,24$ and $48 \mathrm{~h}), 0.5 \mathrm{ml}$ of sample was removed from receptor compartment for spectrophotometric determination and replaced immediately with an equal volume of fresh receptor medium. Samples were analyzed for protein content with Bradford reagent and SOD activity with SOD assay kit. In this experiment, an aqueous solution of SOD with the same concentration was used as control.

\section{Permeation study of SOD loaded niosomes through the synthetic membrane and guinea pig skin}

Wahlberg substantiated the value of guinea pig abdominal skin as hairy and ear skin as a non-hairy animal model for follicular permeation studies [28]. Therefore in this study, permeation studies were performed using hairy and non-hairy guinea pig skin and a synthetic membrane fabricated by Dorsa Behsaz Co (Tehran, Iran). Static Franz diffusion cells were used and hairy and non-hairy skins were mounted between donor and receptor phases. All skin and synthetic membranes provided $3.49 \mathrm{~cm}^{2}$ surface area. Synthetic hair membrane made by epoxy resin, including 90 human hair follicles with $45 \mu \mathrm{m}$ per each $\mathrm{cm}^{2}$ as seen in humans [29]. The donor compartment was filled with SOD-loaded noisome formulations (50 $\mathrm{U} / \mathrm{ml}$ ) while the receptor compartment was filled with $25 \mathrm{ml}$ phosphate buffer ( $\mathrm{pH}$ 7.4). The diffusion cell was placed and clamped in a water bath at $37 \pm 0.5^{\circ} \mathrm{C}$ placed on a magnetic stirrer with a heater. The receptor media were magnetically stirred at 100 $\mathrm{rpm}$. Permeation study extended until 5-folds of lag time that was calculated in the preformulation study (i.e. sampling was continued for $48 \mathrm{~h}$ ). At predetermined time intervals, $2 \mathrm{ml}$ of media was removed from the receptor compartment for spectrophotometric determination and replaced immediately with an equal volume of fresh receptor medium. Samples were analyzed for protein content with Bradford reagent and SOD activity with SOD assay kit. The solution of SOD in buffer phosphate (pH 7.4]) was used as control. Lipids in hairy and non-hairy guinea pig skin were extracted by a method described by Wertz and Downing (first with methanol and then with chloroform/methanol 2:1 at room temperature) [30] and permeability parameters before and after lipid extraction were compared to get clear information about the role of sebum on drug delivery into the follicles.

\section{Statistical methods}

The One-Way (ANOVA) Analysis of Variance and unpaired t-test was used. In addition, to study the relationship between variables and responses, multiple regressions were carried out. The $p$-value of 0.05 and less was considered statistically significant. Data analysis was done with Microsoft Excel and Minitab 16.

\section{RESULTS AND DISCUSSION}

\section{Niosome characterization}

The physicochemical and biopharmaceutical characteristics of niosomes prepared based on factorial experimental design is shown in table 2. A total of eight experimental runs were generated for the evaluation of the main effects and possible interactions. Accordingly, eight formulations were prepared (F1-8).

\section{-Niosome particle size and zeta potential}

Niosome size of freshly prepared formulations varied between 152 $325 \mathrm{~nm}$ and as seen, the smallest and the biggest sizes belong to formulation F1 and F6, respectively. Formulation F1 is made by high levels of S. L/l. L, L/S ratios and Labrafil as surfactant while formulation F6 consisted of S. L/l. L=9 and $\mathrm{L} / \mathrm{S}=20$. Regression analysis of results showed the lowest size was obtained by labrafil and $\mathrm{S}$. $\mathrm{L} / \mathrm{l} . \mathrm{L}=19$. The mathematical model indicated a significant correlation between the surfactant type and S. L/l. L variables and niosome size. The results of ANOVA as shown in table 2 indicated that the model was significant for niosome size.

Drug delivery into the hair follicle depends on different factors such as particle size. Schaefer et al. found that fluorescent polystyrene beads between 5 to $7 \mu \mathrm{m}$ in diameter were deposited deep in the hair follicle whereas smaller or larger beads localized in the stratum corneum and skin surface [31]. Lademann et al. reported that particle sizes between $300-600 \mathrm{~nm}$ penetrate more efficiently and deeper into the hair follicles than non-particulate substances [32]. Also, Vogt et al. found that particles in the size around of $750 \mathrm{~nm}$ remain in the superficial parts of the infundibulum of terminal human hair while particles sized $40 \mathrm{~nm}$ penetrate deeper into the follicular duct [33].

Therefore, it seems that niosomes that were prepared in this study with particle sizes around 150-330 nm increased SOD partitioning and penetration into the hair follicles.

On the other hand, zeta potential values were between-25.3 to-37.7 $\mathrm{mV}$ that mainly affected by the type of surfactant and higher zeta potential was obtained by using SLS as a surfactant. SLS is an anionic surfactant usually a mixture of sodium alkyl sulfate that provides negative charges. Labrafil is a non-ionic surfactant that librates less negative charges compared to SLS. On the other hand, lecithin in $\mathrm{pH}=7.4$ demonstrates a slight negative charge.

\section{- SOD EE\% and activity}

Niosomes illustrated SOD EE\% between 49-90\%, a value well suited for hydrophilic compounds. The correlation between independent variables and EE\% is shown in fig. 1 . The results of ANOVA (table 3) demonstrated the significance of model for EE\%. EE\% was affected by surfactant properties such as hydrophilic-lipophilic balance (HLB). Maximum SOD entrapment provided by Labrafil and SLS was $90 \%$ and $74 \%$, respectively. This difference is significance $(\mathrm{P}=0.011)$ and mainly depend on surfactant HLB. It seems that Labrafil ${ }^{\circledR}$ with $\mathrm{HLB}=4$ in comparison with SLS with $\mathrm{HLB}=40$ increased more SOD solubility in the bilayer membrane. Based on method preparation, multilamellar vesicle (MLV) is probably formed consisting of several lamellar phase lipid bilayers [34]. Although hydrophilic molecules such as SOD mainly are located in the aqueous core, the appropriate surfactant can increase SOD entrapment into the bilayer. That means protein entrapment in MLV is mainly dependent on solubility in the lipid bilayer. Therefore Labrafil ${ }^{\circledR}$ cased higher SOD solubility in bilayer membrane and higher protein entrapment compared to SLS with the hydrophilic property. In this study, two surfactants with completely different HLB have been chosen to articulate the role of surfactant properties on drug loading into the niosomes. Different vehicles such as poly (DL-Lactide-Co-Glycolide) (PLGA) microsphere, alginate-chitosan microsphere [35], cationic niosome [36] and mucoadhesive chitosan-coated niosome [20] have been used as SOD vehicle. The highest and lowest SOD \% entrapments were $92 \%$ and $17 \%$, provided by alginate-chitosan microspheres and cationic niosomes, respectively. The entrapment in chitosancoated niosome $(64 \%)$ was more than cationic niosomes (17\%). It seems that SOD solubility, particle size, SOD-excipients interaction and physical state of carriers play a critical role in determining the extent of entrapment. Microspheres with solid-state provided more SOD entrapment compared with niosomes. In this study, niosomes were prepared by labrafil, provided EE\% equal to alginate-chitosan microspheres. In the other hand, in different studies, liposomes with a similar structure to niosomes have been used for SOD loading and EE\% 20-40\% was reported [37, 38]. It seems that using of suitable surfactant in niosomes, significantly increased SOD EE\% compared with liposomes.

In formulations F4 and F8 immediately after preparation, the activities of SOD loaded in niosomes were $73.3 \%$ and $67.5 \%$, respectively. The activity of SOD in aqueous solution with the same concentration was $84.5 \%$. It can be concluded there was little activity loss during the production procedure. The evaluation of the effect of Triton X-100 on SOD activity demonstrated a decrease in enzyme activity of less than $5 \%$.

\section{In vitro SOD release studies}

In vitro release profiles of SOD from niosomal formulations are shown in fig. 2. The percentage of SOD released after $30 \mathrm{~min}(\% \mathrm{R}-$ $0.5)$ and $48 \mathrm{~h}(\% \mathrm{R}-48)$ were chosen for the evaluation of burst and delayed-release, respectively. Regression analysis of results shows a significant correlation between all independent variables and $\% \mathrm{R}-0.5$ in this manner that higher \%R-0.5 was provided by formulations including Labrafil ${ }^{\circledR}$ as a surfactant, higher S. L/l. L 
and lower L/S ratios. Likewise, \%R-48 was 78.9-91.8\% (formulation F1 and F4, respectively). Higher \%R-48 was obtained in formulations using Labrafil ${ }^{\circledR}$ as a surfactant. Also significant ( $p$ $=0.015$ ) and indirect correlation was found between zeta potential value and \%R-48. Release pattern suggests burst release with all formulations especially F4. This means more than $30 \%$ of the total loaded enzyme released fast after $30 \mathrm{~min}$. Previously, SOD burst release from microspheres has been reported [27]. But SOD didn't show burst release from PLGA and chitosan-alginate microspheres and only $43.7 \%$ of loaded SOD released after $15 \mathrm{~d}$ [35]. This burst release is in accordance with the hydrophilic properties of SOD with high affinity for aqueous bulk phase. Some parts of SOD that incorporated in the aqueous core show a sustained release pattern that previously it was observed for SODloaded microspheres. $70-80 \%$ of enzymes were released from microspheres within the first day, with total release complete by 4-8 d [27]. In comparison between microspheres and niosomes based on the present study, it can be concluded that microspheres presented a more sustained release profile than niosomes. The solids state of microspheres compared with liquid state of niosomes may is the reason for a SOD sustained release profile.

Table 2: Sample sequence from the design of experiments and corresponding niosome size, entrapment efficiency and activity of SOD loaded in niosomes

\begin{tabular}{|c|c|c|c|c|c|c|c|}
\hline Batch & Surfactant & Zeta potential [mv] & (S. L/l. L) ratio & $(\mathrm{L} / \mathrm{S})$ ratio & Mean size [nm] & EE\% & SOD activity [\%] \\
\hline F1 & Lab & -30 & 19 & 20 & $152 \pm 11$ & $59.1 \pm 6.3$ & $68.4 \pm 7.9$ \\
\hline $\mathrm{F} 2$ & SLS & -37.2 & 19 & 20 & $305 \pm 20$ & $75.44 \pm 4.8$ & $71.5 \pm 9.4$ \\
\hline F3 & Lab & -25.3 & 19 & 10 & $190 \pm 8$ & $49.11 \pm 3.5$ & $69.2 \pm 5.8$ \\
\hline $\mathrm{F} 4$ & SLS & -37.7 & 19 & 10 & $244 \pm 16$ & $89.6 \pm 7.1$ & $71.4 \pm 8.3$ \\
\hline F5 & Lab & -28.8 & 9 & 20 & $240 \pm 18$ & $74.5 \pm 8.2$ & $73.3 \pm 8.8$ \\
\hline F6 & SLS & -34.8 & 9 & 20 & $325 \pm 33$ & $82.2 \pm 5.4$ & $70.1 \pm 5.4$ \\
\hline F7 & Lab & -30.5 & 9 & 10 & $290 \pm 19$ & $56.1 \pm 5.5$ & $71.5 \pm 6.6$ \\
\hline F8 & SLS & -33.2 & 9 & 10 & $312 \pm 25$ & $80.1 \pm 6.8$ & $67.5 \pm 7.1$ \\
\hline
\end{tabular}

Values are mean \pm SD $[n=5] .$, Lab,Labrafil®; SLS: Sodium saurylsulphate, S. L/l. L: solid lipid/liquid lipid ratio, EE\%: entrapment efficiency

Table 3: Summary of ANOVA for niosome size and SOD EE [\%] in niosomes prepared by $2^{3}$ factorial design

\begin{tabular}{|c|c|c|c|c|}
\hline Source & F-value & P-value & $\mathbf{r}^{2}$ & $\mathbf{r}^{2}$ [adjust] \\
\hline Niosome size & & & $99.72 \%$ & $99.48 \%$ \\
\hline S/l & 282.4 & 0.001 & & \\
\hline $\mathrm{L} / \mathrm{S}$ & 18.69 & 0.09 & & \\
\hline$S^{\prime}$ & 9.9 & 0.014 & & \\
\hline EE\% & & & $98.94 \%$ & $98.02 \%$ \\
\hline $\mathrm{S} / \mathrm{l}$ & 19.85 & 0.002 & & \\
\hline $\mathrm{L} / \mathrm{S}$ & 61.67 & 0.001 & & \\
\hline $\mathrm{S}$ & 9.14 & 0.016 & & \\
\hline
\end{tabular}

\section{the effect of independent variables on SOD EE\%}

$\mathbf{E E} \%$

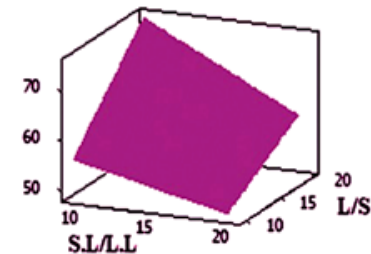

EE\%

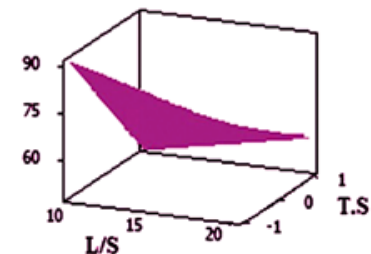

EE\%

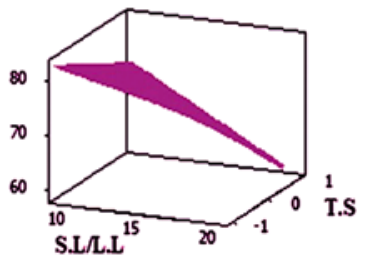

Fig. 1: Surface plots of correlations between independent variables and SOD entrapment efficiency (EE\%) 


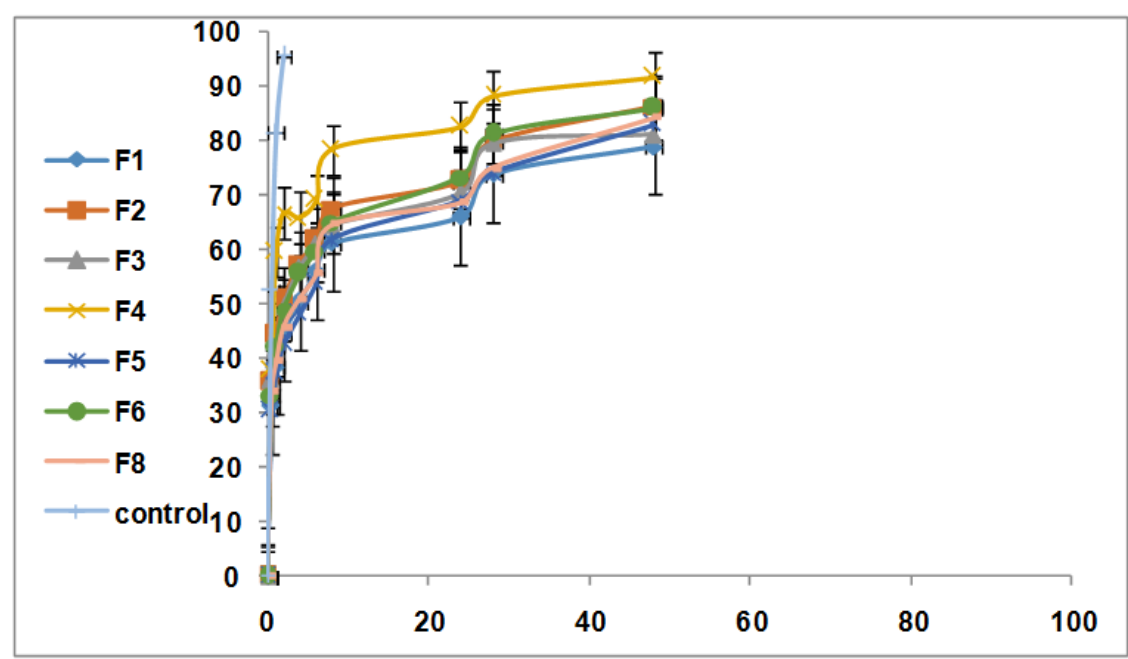

Fig. 2: Release profiles of SOD from niosome formulations F1-8 in phosphate buffer at $\mathrm{pH}$ 7.4. Data are expressed as the mean \pm SD $(n=5)$

\section{SOD-loaded niosome permeation through guinea pig skin and synthetic membrane}

The cumulative activity of SOD permeated across guinea pig skin after 1 (\%A1) and 48 (\%A48) hours are shown in table 4. Amount of protein permeated through hairy and non-hairy guinea pig skin before and after lipid extraction and through the synthetic membrane are presented in fig. 3 All membranes were treated with formulations F1, F8 and SOD solution of the same concentration. F1 and F8 formulations present highest and the lowest amount of independent variables (table 1), respectively. Therefore formulation F1 with low particle size and EE\% together formulation F8 with the high particle size and $\mathrm{EE} \%$ were selected for permeation study. Based on these results, different comparisons were applied that are described below.

\section{- Comparison between hairy and non-hairy guinea pig skin}

The amount of SOD permeated (\%) after $1 \mathrm{~h}\left(\% \mathrm{P}_{1}\right)$ and $48 \mathrm{~h}\left(\% \mathrm{P}_{48}\right)$ across hairy and non-hairy guinea pig skins after application of $\mathrm{F} 1$ and F8 formulations and SOD solution were calculated and compared. The arrangement based on \% 1 and \%P48 presented below:

\%P1: F1 and F8 in hairy skin $>$ SOD solution in hairy skin $>$ F1 and F8 in non-hairy skin $>$ SOD solution in non-hairy

\%P48: F1 and F8 in hairy skin $>$ SOD solution in hairy skin $>$ F1 in nonhairy skin $>$ SOD solution in non-hairy $>$ F8 in non-hairy skin

Based on these results different conclusion can be presented:

$-\% \mathrm{P} 1$ and $\% \mathrm{P} 48$ in hairy skins were significantly bigger for both niosomal formulations (F1 and F8) and free solution in comparison to non-hairy guinea pig skins. Both formulations increased SOD affinity fora follicular pathway that was more than SOD solution and no significant difference was found between F1 and F8. It means that particle size and entrapment ratio were not critical parameters for increasing SOD delivery into the follicles. Therefore, the main route for SOD permeation through guinea pigskin is a follicular pathway that is in accordance with its high molecular weight and hydrophilic nature. The importance of follicular pathway for proteins as hydrophilic high molecular weight compounds has previously been reported [39]. Nano-sized drug delivery systems demonstrate some advantages for drug delivery via the follicles. Nanoparticles can aggregate in the follicular opening and penetrate through the follicle duct and act as a reservoir [40]. The sebaceous glands open into the lower infundibulum of the hair follicle and by sebum secretion create a lipid-enriched environment. Drug penetration and deposition in hair follicles depend on the interaction between a drug and its carrier with sebum [41]. Therefore, the lipid carriers such as niosomes, solid lipid nanoparticle, and nanoemulsions are efficient drug carriers for sebum interaction and accumulation in hair follicles. The lipid state of niosomes facilitates sebum interaction and penetration across the hair follicles [42]. Therefore, to prove the above-mentioned hypothesis, \%P1 and \%P48 after lipid extraction were estimated through hairy and non-hairy guinea pig skins. Results indicated that after lipid extraction \% P1 and \% P48 reduced significantly in comparison with prior to lipid extraction. This means that interaction between niosomes and sebum plays a critical role in SOD accumulation in hair follicles. The reduction in \%P1 and \%P48 after lipid extraction for both niosomal formulations were more than for SOD in solution. In conclusion, niosome formulations increased SOD partitioning into follicles by interaction with sebum.

-The values of $\% \mathrm{P}_{1}$ and $\% \mathrm{P} 48$ for both niosomal formulations were significantly higher than those for SOD solution in hairy skin models. But in non-hairy skin, the value of \%P48 after application of SOD solution was lower than F1 and higher than F8. It means that in nonhairy skin, early hours after application both formulations increased SOD partitioning into the skin more than SOD solution that is in accordance with its hydrophilic property. But after several hours, SOD permeation through non-hairy skin $(\% \mathrm{P} 48)$ was affected by particle size; therefore, F8 with high particle size demonstrated lower values of $\% \mathrm{P} 48$ than SOD solution.

\section{- Comparison between hairy and non-hairy guinea pig with a synthetic membrane}

-Hairy skin: No significant difference was found between \%P1 obtained for SOD solution after application on hairy guinea pig skin and synthetic membrane. However, for $\% \mathrm{P} 48$, this difference was significant with higher values obtained for synthetic membrane. Both niosomal formulations provided more \%P1 and \%P48 in hairy guinea pig skin than synthetic membrane. This finding confirmed the role of sebum in SOD-loaded niosome penetration through the follicular pathway. On the other hand, \%P1 and \%P48 provided after application of both niosomal formulations on hairy guinea pig skin were significantly different from these values obtained for SOD solution. This finding correlates well with results published by Blume-Peytavi et al. [41] who pointed out the role of sebum on drug delivery via hair follicles.

-Non-hairy skin: The permeability of SOD through the synthetic membrane was more than non-hairy guinea pig skin when SOD solution and both niosomal formulations were applied.

-The activity of SOD after application on hairy and non-hairy skins

Both formulations demonstrated more SOD activity after application on guinea pig skins more than free-SOD. But no significant difference was found between hairy and non-hairy guinea pig skins (table 4). 
Previously we reported that solid lipid nanoparticles increased SOD activity in the burned rat skin [43]. It seems that lipid carriers such as niosomes and solid lipid carrier area good carrier for SOD that protects enzyme activity and increases localization into the skin.

Table 4: The enzyme activity of protein permeated through different membranes after 1 and $48 \mathrm{~h}$ [mean \pm SD, $n=5$ ]

\begin{tabular}{llll}
\hline Formulation & \multicolumn{2}{l}{ Hairy guinea pig skin } & Non-hairy guinea pig skin \\
\cline { 2 - 4 } & $\mathbf{0 A}_{\mathbf{1}}$ & $\mathbf{\% A}_{\mathbf{4 8}}$ & $\mathbf{\% A}_{\mathbf{1}}$ \\
\hline Formulation 1 & $20.3 \pm 1.5$ & $68.5 \pm 6.7$ & $15.2 \pm 1.7$ \\
Formulation 8 & $23.2 \pm 2.5$ & $67.9 \pm 5.6$ & $13.3 \pm 1.5$ \\
Free-SOD & ND & $50.2 \pm 3.9$ & $2.5 \pm 0.2$ \\
\hline
\end{tabular}

$\% \mathrm{~A}_{1}=$ enzyme activity due to protein permeated through a membrane after $1 \mathrm{~h}, \% \mathrm{~A}_{48}=$ enzyme activity due to protein permeated through a membrane after $48 \mathrm{~h}$

F1
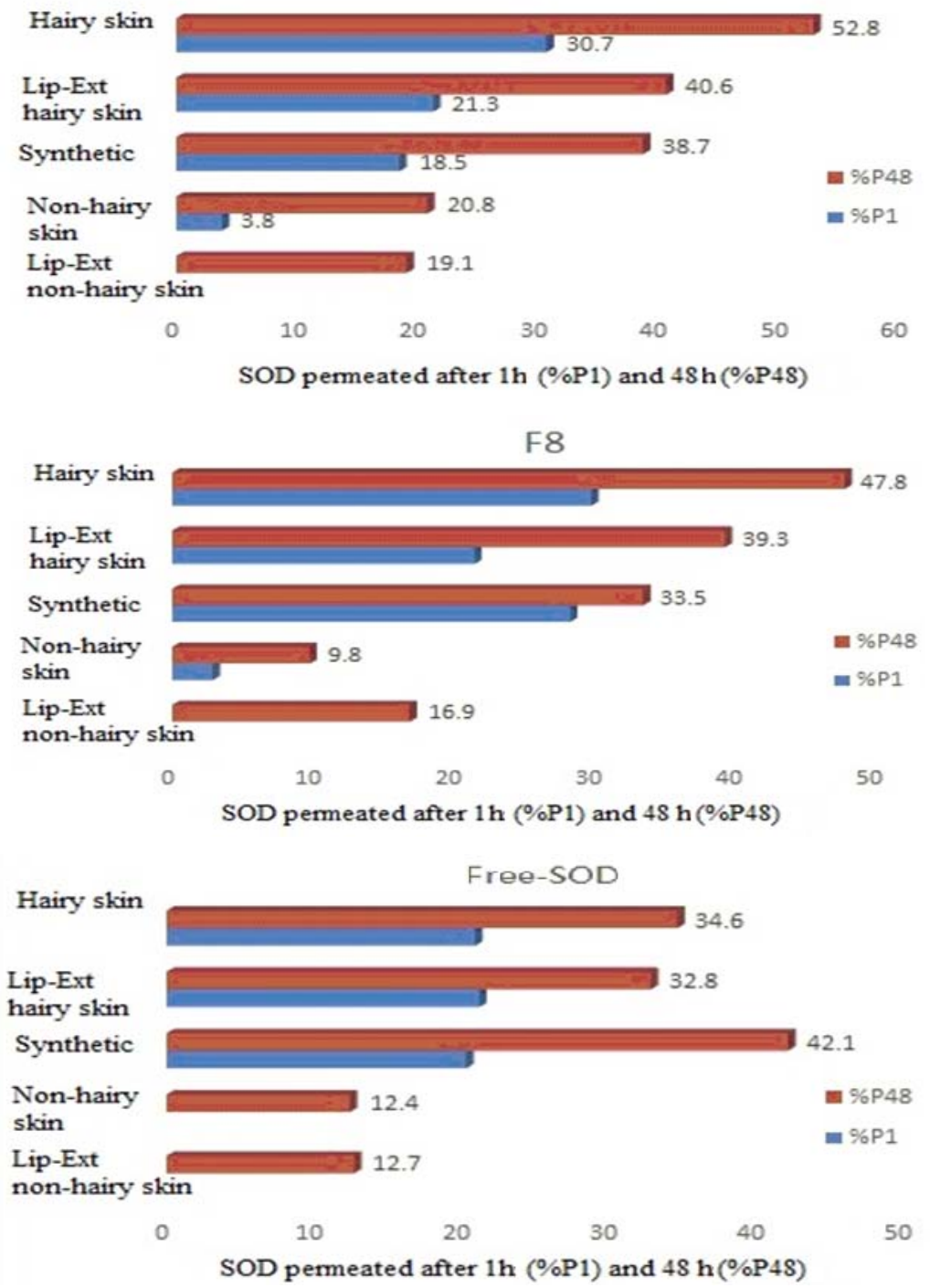

Fig. 3: Permeation of free SOD aqueous solution (Free-SOD) and SOD-loaded in formulations 1 (F1) and 8 (F8) across the hairy skin, lipid extracted hairy skin (Lip-Ext hairy skin), non-hairy skin, lipid extracted non-hairy skin (Lip-Ext non-hairy skin), synthetic membrane

(Synthetic)

\section{Niosome stability}

Niosome size, SOD content, SOD activity, and dispersion appearance were evaluated after $6 \mathrm{mo}$ of storage at $2-8^{\circ} \mathrm{C}$ as indicators of formulation stability. Fig. 4 shows the AFM picture of niosome suspension immediately after preparation (fig. $4 \mathrm{~A}$ ) and six months of storage at $2-8^{\circ} \mathrm{C}$ (fig. B). Results indicated no significant particle size growth. Niosomes in both cases were spherical with a little extension on the surface that previously has been attributed to drug crystals on the surface of niosomes [44]. Moreover, after six months SOD content and activity decreased less than $5 \%$ and dispersion appearance did not change. Therefore, the prepared niosomal formulations were characterized as stable dispersions that protected the enzyme from degradation. Previously, Manca et al. evaluated the vesicle stability by AFM visualization technique and particle size analyzer $[45,46]$. 

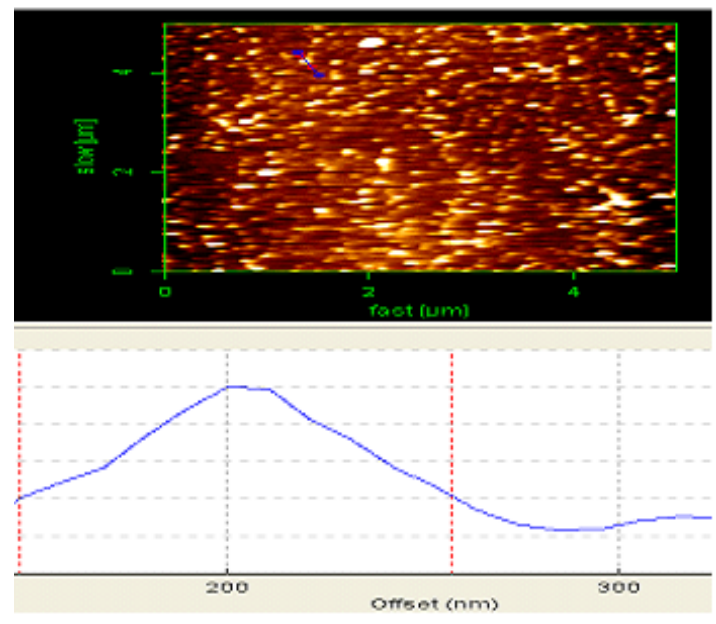

8

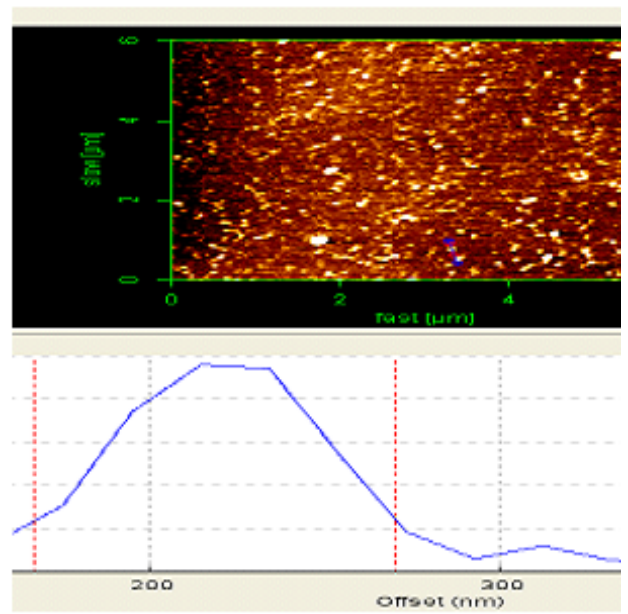

Fig. 4: AFM images of niosomal formulation immediately after preparation (A) and after 6 mo of storage at $2-8^{\circ} \mathrm{C}(\mathrm{B})$

\section{CONCLUSION}

Niosomes demonstrated stable vehicle for SOD loading that preserved enzyme activity. More than $90 \%$ of total SOD was encapsulated into the niosomes made by labrafil as a non-ionic surfactant. Type of surfactant played a critical role in niosomes characters. Niosomes prepared by labrafil indicated lower particle size and zeta potential that niosomes made by SLS as an anionic surfactant. SOD permeated through the skin mainly by a follicular pathway that niosomes made by both surfactants increased the affinity of SOD for the follicular pathway and induce high enzyme activity across the hair shaft. Niosome low particle size and interaction with the sebum were the main reason for increasing SOD affinity to follicular root. In conclusion, niosomes illustrated good properties as SOD follicular delivery systems that protected SOD against degradation and increased enzyme affinity to follicular root that is the site of action in alopecia aretea localization in the site of action.

\section{ACKNOWLEDGMENT}

This study was carried out under financial support provided by Ahvaz Jundishapur University of Medical Sciences (AJUMS), so here we thank vice chancellor for research and technology of AJUMS. The authors are thankful for Iranian Representation for Gattefosse Pharmaceuticals (Faratin Company).

\section{AUTHORS CONTRIBUTIONS}

All the authors have contributed equally

\section{CONFLICTS OF INTERESTS}

Declared none

\section{REFERENCES}

1. Reynolds AJ, Jahoda CA. Inductive properties of hair follicle cells. Annals New York Acad Sci 1991;642:226-41.

2. Sundberg JP, King LE, Basco C. Animal models for the male pattern (androgenetic) alopecia. Eur J Dermatol 2001;11:321-5.

3. Akar A, Arca E, Erbil H, Akay C, Sayal A, Gur AR. Antioxidant enzymes and lipid peroxidation in the scalp of patients with alopecia areata. J Dermatol Sci 2002;29:85-90.

4. Zafar R, Abdullateef A, Alzolibani B, Hani A, Al-Shobaili B, Saif $\mathrm{GB}$, et al. Biochemical and immunological studies on erythrocytes superoxide dismutase modified by nitric oxide in patients with alopecia areata: Implications in alopecia patchy persistent and alopecia universalis. Immunol Lett 2014;160:50-7. https://doi.org/10.1016/j.imlet.2014.03.007
5. Alzolibani AA. Preferential recognition of hydroxyl radicalmodified superoxide dismutase by circulating autoantibodies in patients with alopecia areata. Ann Dermatol 2014;25:576-83.

6. Trüeb RM. The impact of oxidative stress on the hair. Int J Cosmet Sci 2015;37:25-30.

7. Chitra KP, Pillai KS. Antioxidants in Health. Ind J Physiol Pharmacol 2002;46:1-5.

8. Seiberg M. Age-induced hair graying. The multiple effects of oxidative stress. Int J Cosmet Sci 2013;35:532-8.

9. Patzelt A, Knorr F, Blume-Pytavi U, Sterry W, Lademann J. Hair follicles, their disorder, and their opportunities. Drug Discovery Today: Disease Mechanisms 2008;5:173-81.

10. Fernandes B, Silva R, Ribeiro A, Matamo T, Gomes AC. Improved poly (D, L-Lactide) nanoparticles. The based formulation for hair follicle targeting. Int J Cosmet Sci 2015;32:282-94.

11. Emerit I, Filipe P, Freitas J. Protective effect of superoxide dismutase against hair graying in a mouse model. Photochem Photobiol 2004;80:579-82.

12. Diehl C. A novel efficient and safe treatment for atopic dermatitis: topical superoxide dismutase. Dermatol Res Skin Care 2017;1:1-7.

13. Manca ML, Manconi M, Nacher A, Carbone C, Valenti D, Maccioni AM, et al. Development of novel diolein-niosomes for cutaneous delivery of tretinoin: influence of formulation and in vitro assessment. Int J Pharm 2014;30:176-86.

14. Manosroi A, Ruksiriwanich W, Abe M, Manosroi J. Transfollicular enhancement of gel containing cationic niosomes loaded with unsaturated fatty acids in rice (Oryza sativa) bran semi-purified fraction. Eur J Pharm Biopharm 2012;18:303-13.

15. Mohawed O, Ashmonny MM, Elgazayerly ON. Nisomeencapsulated clomipramine for transdermal controlled delivery. Int J Pharm Pharm Sci 2014;6:567-75.

16. Acharya A, Kumar K, Ahmed MG, Paudel S. A novel approach to increase the bioavailability of candesartan cilexetil by proniosomal gel formulation in vitro evaluation. Int J Pharm Pharm Sci 2016;8:241-6.

17. Marianecci C, Di marzio L, Rinaldi F, Esposito S, Carafa M. Niosomes from the 80s to present: state of the art. Adv Colloid Interface Sci 2014;205:187-206.

18. Uchegbu IF, Florence AT. Non-ionic surfactant vesicles (niosomes): physical and pharmaceutical chemistry. Adv Collid Interface Sci 1995;58:1-55.

19. Tabbakhian M, Tavakoli N, Jaafari MR, Daneshamouz S Enhancement of follicular delivery of finasteride by liposomes and niosomes: 1 . In vitro permeation and in vivo deposition 
studies using hamster flank and ear models. Int J Pharm 2006:323;1-10.

20. Muhammad F, Brooks JD, Rivirere JE. Comparative mixture effects of JP-8 additives on the dermal absorption and disposition of jet fuel hydrocarbons in different membrane model systems. Toxicol Lett 2004;150:351-65.

21. Simon GA, Maibach HI. Relevance of hair lost mouse as an experimental model of percutaneous penetration in man. Skin Pharmacol Appl Skin Physiol 1998;11:80-6.

22. Anderson KE, Maibach HI, Anjo MD. The guinea pig: an animal model for human skin absorption of hydrocortisone, testosterone and benzoic acid? Br J Dermatol 1980;102:447-53

23. Sharif Makhmal Zadeh B, Moghimi HR, Santos P, Lane ME, Hadgraft J. Comparative study of the in vitro permeation characteristic of sulphadiazine across synthetic membranes and eschar tissue. Int Wound J 2008;5:633-8.

24. Abed E, Yousef SA, Pastore MN, Telaprolu K, Mohammed YH, Namjoshi S, et al. Skin models for the testing of transdermal drugs. Clin Pharmacol 2016;8:163-76.

25. Bangham A, De Gie J. Osmotic properties and water permeability of phospholipid liquid crystals. Chem Phys Lipid 1967; 1:225-31.

26. Rengel RG, Barisic K, Pavelic Z, Grubisic TZ, Cepelak Z, Filipovic Grcic J. High-efficiency entrapment of superoxide dismutase into mucoadhesive chitosan-coated niosomes. Eur J Pharm Sci 2002;15:441-8.

27. Celik 0, Akbuga J. Preparation of superoxide dismutase loaded chitosan microspheres: characterization and release studies. Eur J Pharm Biopharm 2007;66:42-7.

28. Wahlberg JE. Transepidermal or transfollicular absorption in vivo and in vitro studies in hairy and non-hairy guinea pig skin with sodium and mercuric chlorides. Acta Derm Venereol 1968;48:336-44.

29. Otberg N, Richter H, Schaefer H, Blume-Peytavi U, Sterry W, Lademann J. Variation of hair follicle size and distribution in different body sites. J Invest Dermatol 2004;122:14-9.

30. Wertz PW, Downing DT. Integral lipids of human hair. Lipids 1988;23:878-81.

31. Schaefer H, Watts F, Brod J, Hel JB. Follicular penetration. In: RC Scott, RH Guy, J Hadgraft. (editors). Prediction of percutaneous penetration, methods, measurement, and modeling. IBC Technical services, London; 2003. p. 63-173.

32. Lademann J, Richte H, Teichmann A, Otberg N, Blume Peytavi U, Luengo J, et al. Nanoparticles-an efficient carrier for drug delivery into the hair follicles. Eur J Pharm Biopharm 2007;66:159-64.

33. Voget A, Combadiere B, Hadam S, Stieler KM, Lademann J, Schaefer $\mathrm{H}$, et al. $40 \mathrm{~nm}$ but not $750 \mathrm{~nm}$ or $1500 \mathrm{~nm}$, nanoparticles enter epidermal CD1cells after transcutaneous application on human skin. J Invest Dermatol 2006;126:131622.

34. Patil PY, Jadhav S. Novel methods for liposome preparation. Chem Phys Lipids 2014;177:8-18.

35. Wang YE, Gao GQ, Zheng CH, XU DH, Liang WQ. Biodegradable and complex microspheres used for sustained delivery and activity protection of SOD. J Biomed Material Res Part B: Appl Biomat 2006;27:74-8.

36. Kigasawa K, Miyashita M, Kajimoto K, Kanamura K, Harashima $\mathrm{H}$, Kogure K. Efficient intra-dermal delivery of superoxide dismutase using a combination of niosomes and iontophoresis for protection against UV-Induced skin damaged. Biol Pharm Bull 2012;35:781-5.

37. Corvo ML, Jorge JCS, Vanthof R, Cruz M, Crommolin JA, Strom G. Superoxide dismutase entrapped in long-circulating liposomes: formulation design and therapeutic activity in rat adjuvant arthritis. Biochem Biophys Acta 2002;1564:227-36.

38. Porfire AS, Tomuta I, Leucuta SR, Achim M. Superoxide dismutase loaded liposomes. The influence of formulation factors on enzyme encapsulation and release. Farmacia 2013;61:865-73.

39. Mitragotri S. Modeling skin permeability to hydrophilic and hydrophobic solutes based on four permeation pathways. J Controlled Release 2003;86:69-92.

40. Schafer Korting M, Mehnert W, Korting HC. Lipid nanoparticles for improved topical application of drugs for skin diseases. Adv Drug Delivery Rev 2007;59:427-43.

41. Blume Peytavi U, Vogt A. Human hair follicle: reservoir function and selective targeting. Br J Dermatol 2011;165:13-7.

42. Betz G, Imboden R, Imboden G. Interaction of niosome formulation with human skin in vitro. Int J Pharm 2001;229:117-29.

43. Karami MA, Sharif Makhmal Zadeh B, Kouchak M, Moghimipur E. Superoxide dismutase loaded solid lipid nanoparticles prepared by cold homogenization method: characterization and permeation study through burned rat skin. Jundishapur J Natur Pharm Prod 2016;11. Doi:10.17795/jjnpp-33968

44. Toongsuwan S, Li LC, Erickson BK, Chang HC. Formulation and characterization of bupivacaine lipospheres. Int J Pharm 2004;280:57-65.

45. Manca ML, Castangia I, Zaru M, Nacher A, Valenti D, Fernandez Busquets X. Development of curcumin loaded sodium hyaluronate immobilized vesicles (hyalurosomes) and their potential on skin inflammation and wound restoring. Biomat 2015;77:100-9.

46. Manca ML, Castangia I, Matricardi P, Lampis S, Fernandez Busquets X, Fadda AM. Molecular arrangements and interconnected bilayer formation induced by alcohol or polyalcohol in phospholipid vesicles. Colloids Surface B: Biointerfaces 2014;117:360-7. 